\title{
El fair play, la victoria y la cuestión de género en el fútbol en el ámbito de la educación primaria
}

\section{The fair play, the victory and the gender issue in football in the context of primary education}

\author{
María Cabañero García* y Roberto Velázquez Buendía \\ Universidad Autónoma de Madrid. Facultad de Formación del Profesorado y Educación. \\ Departamento de Educación Física, Deporte y Motricidad Humana. Madrid (España).
}

\begin{abstract}
Resumen: El fútbol es un fenómeno social, cultural, mediático y económico. Existen numerosas investigaciones sobre su influencia en quiénes lo practican. No obstante, el objetivo de esta investigación fue explorar su influencia sobre valores sociales relacionados con el fair play, la igualdad de género y la relación medios-fines, en alumnado de primaria, por ser aspectos que pueden reflejarse en algunas problemáticas sociales. La población objeto de estudio fue alumnado de sexto curso. Es un estudio descriptivo correlacional transversal. Las fuentes de obtención de información utilizadas fueron, un cuestionario y un grupo de discusión. Los resultados indicaron que el sexo y la práctica de fútbol federado influyen en el fair play, afán por la victoria e igualdad de género, siendo los chicos y el alumnado practicante de fútbol más proclive a conductas anti fair play, más resultadistas y con una visión más negativa hacia la figura de la mujer en el fútbol.

Palabras de clave: Fútbol, fair play, victoria, mujer, valores.
\end{abstract}

Abstract: Football is a social, cultural, mediatic and economic phenomenon. There is a great deal of research on its influence on who practices it. However, the objective of this research was to explore its influence on social values related to fair play, gender equality and the means-ends relation, in elementary students, as aspects that can be reflected in some social problems. The study population was students of sixth grade. It is a cross-sectional descriptive study. The sources of information used were a questionnaire and a discussion group. The results indicated that gender and federated soccer practice influence fair play, a desire for victory and gender equality, being boys and students practicing soccer more prone to anti-fair behavior, more result-focused and with a more negative view towards the figure of women in football.

Keywords: Football, fair play, victory, woman, values.

\section{Introducción}

Al encender la televisión, en la mayoría de canales y a cualquier hora, son constantes las noticias que hablan de conflictos, fraude, discriminación o violencia de género. Éstas son algunas de las principales problemáticas sociales de la actualidad en el mundo y en España. De un lado, algunas situaciones vividas en nuestro país, como la elevada tasa de desempleo, los desahucios, los recortes sociales, o la más que normalizada y tan de moda corrupción política que goza de gran respaldo, provocan numerosas protestas y conflictos a diario. Del otro, la discriminación de la mujer, que por tradición histórica siempre ha estado relegada a un segundo plano socio-político y económico y sometida en un mundo androcéntrico, provoca en muchos casos, episodios de violencia de género en los que un alto porcentaje de mujeres muere a manos de sus parejas. Todos estos aspectos provocan la desconfianza del pueblo hacia el sistema y hacia las propias personas, lo que degenera en una crisis de valores como la que se vive actualmente, donde cada vez menos parecen tener cabida valores como el respeto, la honestidad o la solidaridad.

Dirección para correspondencia [Correspodence address]: María Cabañero García. Calle Don Felipe 6, CP 28004, Madrid (España). E-mail: mcaba55@gmail.com
Ante esto, parece necesario inculcar a las generaciones venideras un espíritu crítico, esto es, fomentar en los niños y niñas la capacidad de pensar por sí mismos y analizar la realidad razonando, en un entorno, como vemos, lleno de problemas, para que puedan desarrollar valores necesarios para la convivencia en el seno de una sociedad democrática, como el respeto o la tolerancia, hacia las cosas o actitudes que realmente lo merecen.

Como bien es sabido, el deporte, además del aprendizaje físico y técnico que conlleva, puede ser un medio idóneo para inculcar a los niños y nińas una serie de valores sociales y personales positivos, actitudes y hábitos de comportamiento, mediante una práctica bien organizada (Beregüi y Garcés de los Fayos, 2007; López et al, 2017, 2018), ya que como bien expone Gutiérrez San Martín (1998), la simple práctica deportiva, por sí misma, no genera valores positivos por lo que deben adoptarse una serie de medidas que lo faciliten.

Se sabe que el deporte en general y el fútbol en particular, en el que se centra este estudio, ejercen una gran influencia, sobre todo en los más jóvenes, quienes lo consumen no sólo en la escuela, sino a través de los medios de comunicación, donde, como afirma Ginesta (2007), los valores que se transmiten pueden ser distintos a los que el profesorado intenta in- 
culcar en su labor docente, si es que intenta inculcar algunos, ya que las propias situaciones educativas que ofrece el deporte no surten efecto automáticamente en la moral del alumnado, sino que es necesario darle una intencionalidad por parte del docente (Gil-Madrona, 2006).

Este estudio se centra pues en el deporte del fútbol, ya que por su condición de deporte rey, sería interesante conocer la influencia que ejerce sobre la formación de valores en niños y niñas. En virtud de lo expuesto con anterioridad, el objetivo general de la investigación se centra en explorar la influencia del fútbol en la formación y desarrollo de valores sociales relacionados con el fair play, la igualdad de género y la relación medios-fines en la práctica del fútbol, en los niños y niñas en Educación Primaria. Este objetivo general puede desglosarse en los siguientes objetivos específicos:

- Conocer la visión que el alumnado tiene del fair play en el fútbol.

- Conocer la importancia de la victoria en el fútbol y los fines utilizados, para el alumnado.

- Conocer la opinión que el alumnado tiene sobre la participación de la mujer en el fútbol.

- Comprobar si la práctica de fútbol federado en el alumnado en el influye en la visión que tienen sobre el fair play, el papel de la mujer en el fútbol o la importancia que se le otorga a la victoria.

Se ha escogido hablar de estos tres aspectos, debido a la gran relación que guardan con algunos de los problemas sociales actuales que acabamos de comentar, como la discriminación o el fraude y la búsqueda del éxito a toda costa, considerándose pues estos problemas, siguiendo a Sánchez-Oliva, Leo, Sánchez-Miguel, Amado, y García-Calvo, (2012), antecedentes importantes en la realización de conductas negativas o antisociales.

\section{Método}

\section{Muestra}

La muestra estuvo compuesta por 53 estudiantes de sexto de Primaria, en tres colegios públicos de la Comunidad de Madrid. La muestra se seleccionó de forma no aleatoria por muestreo incidental. En nuestro caso, debido a la naturaleza de los centros donde se ha llevado a cabo la selección de la muestra (centros de una sola línea) y a los problemas con los permisos que permitieran al alumnado participar en la investigación, la representatividad de la muestra es escasa, por lo que se asumen las limitaciones oportunas a la hora de poder generalizar los resultados hallados a toda la población.

SPORT TK: Revista Euroamericana de Ciencias del Deporte

\section{Diseño}

Es un estudio descriptivo correlacional transversal. Las variables se miden y operativizan de forma numérica, por lo que se puede definir según Tejero (2014) como cuantitativo. Aunque debido a la naturaleza de la investigación, es necesario el uso complementario de técnicas cualitativas, como el grupo de discusión, para conocer mejor la opinión del alumnado respecto al objeto de estudio.

Para la elaboración del cuestionario, se modificó un cuestionario validado de evaluación del fair play en futbolistas jóvenes (Boixadós y Cruz, 1995; citado enCruz, Boixadós, Torregrosa y Mimbrero, 1996), y se adaptó de acuerdo a los objetivos de este estudio, y a la edad de los participantes. El cuestionario, de tipo Likert, contempla cuatro opciones de respuesta, desde totalmente de acuerdo (4) a nada de acuerdo (1), y está compuesto por 24 ítems (del 1 al 5 datos del encuestado, y del 6 al 24 preguntas relativas a los tres ámbitos de estudio) que pretenden indagar en la visión que el alumnado tiene del fútbol.Antes de comenzar con la recogida de información, se pasó el cuestionario a un grupo piloto y se realizó un análisis de consistencia interna del instrumento (Alfa de Cronbach), cuyo resultado fue de 0,771 , cifra cercana a 1 , lo que indica una fiabilidad alta del instrumento.

Por lo que respecta al grupo de discusión, se constituyeron dos, formados por 7 y 6 participantes, respectivamente, "cantidad situada entre los márgenes recomendados [...] por especialistas en esta técnica de investigación” (Ibañez, 1979; Ortí, 1986; citado en Velázquez y Hernández, 2002, p.372). La duración de funcionamiento de los mismos fue de aproximadamente 50 minutos cada uno. Los grupos de discusión contaron con la presencia de la investigadora que hizo las funciones de moderadora.

En cuanto a las variables que se han trabajado en el estudio, según el enfoque metodológico de la investigación (Bisquerra, 2004), se pueden diferenciar:

- Variables discriminativas o de segmentación: En este caso fue el sexo. La muestra estuvo compuesta por 27 chicos $(50,94 \%)$ y 26 chicas $(49,05 \%)$.

- Variables correlacionadas y predictoras (constantes): Fueron, por un lado, la práctica de fútbol en un equipo federado en horario extraescolar, y, por otra, el tiempo de experiencia.

\section{Tratamiento de los datos}

- Datos procedentes del cuestionario

El análisis estadístico de los resultados se centró en el estudio de la distribución de frecuencias para el total de la muestra y para las variables sexo y práctica extraescolar de fútbol, dado el carácter descriptivo del cuestionario. 
- Datos procedentes del grupo de discusión

Se realizó una transcripción literal de las grabaciones de lo acontecido en los grupos de discusión, incorporando las anotaciones tomadas por la investigadora-observadora durante el debate. Se establecieron tres categorías con sus correspondientes subcategorías para el análisis del discurso:

1. Juego limpio: Dureza, entrenador/a, arbitraje, acciones ilegales y comparación con otros deportes.

2. Victoria: Satisfacción, justicia y éxito.

3. Mujer: Acuerdo, sexismo, calidad, estética y comparación con fútbol masculino.

Después, se cruzaron los datos y posteriormente se analizó la información obtenida a través de cada grupo de discusión estableciendo las conclusiones correspondientes y relacionando los resultados aquí obtenidos de forma cualitativa, mediante el programa Atlas ti 6.0., con los datos cuantitativos obtenidos del programa estadístico SPSS v.21.0.

\section{Procedimiento}

En primer lugar, se solicitaron las colaboraciones a los centros educativos, los permisos a las familias y se elaboraron los instrumentos de investigación. A continuación, se realizó el trabajo de campo mediante los cuestionarios y grupos de discusión. Finalmente, se analizaron los resultados, se obtuvieron las conclusiones y se realizó el informe final.

Esta investigación se basó en los principios marcados por la comisión ética de la Universidad Autónoma de Madrid en su código de buenas prácticas en investigación elaborado por el Comité de Ética de la Investigación de dicha Universidad y aprobado en Consejo de Gobierno de 8 de febrero de 2013, en cuanto al tratamiento y forma de llevar a cabo un estudio con personas (http://www.uam.es/otros/ceiuam/).

\section{Resultados}

Para determinar la fiabilidad del cuestionario se realizó la prueba de Alpha de Cronbach. El cuestionario muestra unos altos niveles de fiabilidad total $(0.852)$ y por categorías, siendo las categorías de juego limpio y afán por la victoria las que muestran una mayor fiabilidad $(0,690$ y 0.819 , respectivamente), por encima de la categoría de igualdad de género (0.521).

Después y como se observa en la tabla 1, se obtuvieron los estadísticos descriptivos por cada una de las tres categorías en las que habíamos dividido el cuestionario. Según los valores de acuerdo de 1 a 4 que se establecieron en el cuestionario, podemos observar que el grupo tiene actitudes bastante favorables hacia jugar limpio y la igualdad de género en el fútbol (con puntajes superiores a 3), pero muestran un mayor afán por la victoria (puntajes en torno a 2):
Tabla 1. Estadísticos descriptivos de las tres categorías.

\begin{tabular}{llccc}
\hline & & $\begin{array}{c}\text { Juego } \\
\text { Limpio }\end{array}$ & $\begin{array}{c}\text { Igualdad } \\
\text { Género }\end{array}$ & $\begin{array}{c}\text { Afán } \\
\text { Victoria }\end{array}$ \\
\hline \multirow{2}{*}{$\mathrm{N}$} & Válidos & 53 & 53 & 53 \\
\cline { 2 - 5 } & Perdidos & 0 & 0 & 0 \\
\hline Media & 3,0429 & 3,4088 & 2,7278 \\
\hline Mediana & 3,0909 & 3,5000 & 2,7143 \\
\hline Moda & 3,09 & 4,00 & 3,43 \\
\hline
\end{tabular}

Mediante la prueba U de Mann Whitney, se obtuvo la significación entre las respuestas de los ítems según las variables mencionadas anteriormente. En las tablas 2 y 3 sólo se muestran los ítems con diferencias estadísticamente significativas:

Tabla 2. Ítems con diferencias estadísticamente significativas según sexo.

\begin{tabular}{lcccc}
\hline & $\begin{array}{c}\text { U de Mann- } \\
\text { Whitney }\end{array}$ & $\begin{array}{c}\text { W de } \\
\text { Wilcoxon }\end{array}$ & Z & $\begin{array}{c}\text { Sig. asintót. } \\
\text { (bilateral) }\end{array}$ \\
\hline Ítem7 & 151 & 476 & $-3,579$ & 0,001 \\
\hline Ítem8 & 250,5 & 575,5 & $-1,884$ & 0,06 \\
\hline Ítem10 & 149 & 500 & $-3,79$ & 0,001 \\
\hline Ítem13 & 175 & 500 & $-3,101$ & 0,002 \\
\hline Ítem14 & 233,5 & 584,5 & $-2,372$ & 0,018 \\
\hline Ítem17 & 241,5 & 592,5 & $-2,219$ & 0,026 \\
\hline Ítem18 & 201 & 526 & $-2,645$ & 0,008 \\
\hline Ítem20 & 214,5 & 565,5 & $-2,95$ & 0,003 \\
\hline Ítem23 & 196,5 & 547,5 & $-2,897$ & 0,004 \\
\hline
\end{tabular}

Tabla 3. Ítems con diferencias estadísticamente significativas según práctica fútbol federado

\begin{tabular}{lcccc}
\hline & $\begin{array}{c}\text { U de Mann- } \\
\text { Whitney }\end{array}$ & $\begin{array}{c}\text { W de } \\
\text { Wilcoxon }\end{array}$ & Z & $\begin{array}{c}\text { Sig. asintót. } \\
\text { (bilateral) }\end{array}$ \\
\hline Ítem 7 & 162,000 & 792,000 & $-2,770$ & 0,006 \\
\hline Ítem 8 & 202,500 & 832,500 & $-2,191$ & 0,028 \\
\hline Ítem 10 & 108,500 & 774,500 & $-3,969$ & 0,000 \\
\hline Ítem 13 & 110,000 & 740,000 & $-3,811$ & 0,000 \\
\hline Ítem 18 & 177,000 & 807,000 & $-2,487$ & 0,013 \\
\hline Ítem 20 & 212,000 & 878,000 & $-2,176$ & 0,030 \\
\hline Ítem 23 & 199,000 & 865,000 & $-2,149$ & 0,032 \\
\hline
\end{tabular}

A continuación, se expondrán los resultados referentes a cada una de las tres categorías establecidas para el estudio, y a su vez, dentro de estos subapartados se hablará primero de los resultados generales y después se hará lo propio dividiendo 
la muestra por sexo y por el criterio de juega o no a fútbol federado.

\section{Resultados sobre fair play}

A) Resultados totales

Se aprecia una actitud general favorable hacia jugar limpio en el fútbol, especialmente cuando se pregunta sobre contacto físico o reacciones violentas. Se muestran más permisivos cuando se les habla de protestas hacia el árbitro o de jugar duro como respuesta al juego duro del rival, como se puede observar en los porcentajes de respuesta a preguntas como "está bien que un jugador proteste al árbitro sus decisiones" (acuerdo del $49 \%$ ), o "jugar duro se acepta si el otro equipo también juega duro" (acuerdo del 39,6 \%). Se pueden observar datos preocupantes, por ejemplo, en las respuestas al ítem 9 (jugar duro justificado por las exigencias del público) en el cual buena parte del alumnado (19,2\%) justifica de algún modo la violencia, o el ítem 17 en el que un 20,7 \% opina que cualquier forma es válida para detener a un contrario a punto de meter gol.

Tabla 4. Porcentajes de respuesta generales para la categoría juego limpio.

\begin{tabular}{|c|c|c|c|c|}
\hline ¿En qué grado estás de acuerdo con que...? & Nada & Poco & Bastante & Totalmente \\
\hline Ítem 6. Está bien que un jugador/a reaccione violentamente cuando le hacen una falta & $71,7 \%$ & $20,8 \%$ & $7,5 \%$ & $0 \%$ \\
\hline Ítem 9. Jugar duro está justificado si el público anima a hacerlo & $59,6 \%$ & $21,2 \%$ & $9,6 \%$ & $9,6 \%$ \\
\hline Ítem 12. Está bien que un jugador/a proteste al árbitro sus decisiones & $24,5 \%$ & $26,4 \%$ & $22,6 \%$ & $26,4 \%$ \\
\hline Ítem 15. Está bien que un entrenador/a anime a lesionar a un jugador del equipo contrario & $84,9 \%$ & $11,3 \%$ & $1,9 \%$ & $1,9 \%$ \\
\hline $\begin{array}{l}\text { Ítem 17. Un contrario/a que está a punto de marcar gol tiene que ser detenido/a de cualquier } \\
\text { forma, legalmente o no }\end{array}$ & $60,4 \%$ & $18,9 \%$ & $7,5 \%$ & $13,2 \%$ \\
\hline Ítem 20. En los partidos importantes todo está permitido para poder ganar & $67,9 \%$ & $20,8 \%$ & $5,7 \%$ & $5,7 \%$ \\
\hline Ítem 22. Jugar duro se acepta si el otro equipo también juega duro & $34 \%$ & $16,4 \%$ & $15,1 \%$ & $24,5 \%$ \\
\hline Ítem 24. Un jugador/a hábil debe ser parado siempre de forma limpia & $13,2 \%$ & $13,2 \%$ & $20,8 \%$ & $52,8 \%$ \\
\hline Ítem 25. Los oponentes rápidos tiene que ser detenidos de cualquier manera, legalmente o no & $49,1 \%$ & $30,2 \%$ & $11,3 \%$ & $9,4 \%$ \\
\hline Ítem 26. El entrenador/a debería prohibir cometer entradas duras en los partidos & $26,4 \%$ & $11,3 \%$ & $20,8 \%$ & $41,5 \%$ \\
\hline $\begin{array}{l}\text { Ítem 27. Está bien que un equipo intente molestar o enfadar a un jugador/a impulsivo del } \\
\text { equipo contrario }\end{array}$ & $71,7 \%$ & $18,9 \%$ & $1,9 \%$ & $7,5 \%$ \\
\hline
\end{tabular}

Estos resultados se han visto confirmados con lo extraído del análisis de los grupos de discusión, donde, en principio, ninguno de los entrevistados ni entrevistadas catalogó como juego duro las agresiones verbales o las conductas antideportivas como los insultos (únicamente se referían al juego sucio en términos de patadas, codazos, agarrones...). Esta puede ser una de las razones por las cuales la mayoría muestra una actitud más de acuerdo hacia la protesta, ya que no la encuadran dentro de la deportividad.

Además, cabe destacar la visión negativa que la mayoría de los encuestados tienen hacia la figura del árbitro, considerando que no es neutro en la mayoría de los casos (se referían a ello como "van con el otro equipo"), así, en muchas ocasiones, justifican en cierta manera las conductas antideportivas de los jugadores alegando que es normal que se quejen a los árbitros si éstos les están perjudicando.
B) Resultados según sexo

Tanto chicos como chicas muestran actitudes de acuerdo hacia la deportividad y el fair play, sobre todo y como hemos dicho, cuando se habla de acciones físicas, no tanto así cuando se habla de recriminaciones al árbitro, ámbito en el que más de la mitad de los chicos se muestra a favor $(59,3 \%$ frente al $38,5 \%$ de chicas).

No obstante, como mostraba la tabla 2, existen diferencias estadísticamente significativas $(p<0.05)$ entre chicos $y$ chicas en lo referente al fair play y el fútbol, concretamente en lo que a las actitudes antirreglamentarias para detener a un contrario a punto de marcar gol se refiere (ítem 17). Prácticamente un $30 \%$ de los niños dijeron estar de acuerdo, frente a un $11,5 \%$ de las niñas.

Además, en algunas ocasiones la diferencia entre los porcentajes de respuesta de acuerdo y desacuerdo entre niños y niñas son bastante importantes, como se refleja en la siguiente tabla (ítems 9, 12 y 24): 
Tabla 5. Porcentajes de respuesta por sexos para la categoría juego limpio.

\begin{tabular}{|c|c|c|c|c|c|c|c|c|}
\hline \multirow{2}{*}{ ¿En qué grado estás de acuerdo con que...?? } & \multicolumn{4}{|c|}{ Chicos } & \multicolumn{4}{|c|}{ Chicas } \\
\hline & Nada & Poco & Bastante & Total & Nada & Poco & Bastante & Total \\
\hline $\begin{array}{l}\text { Ítem 6. Está bien que un jugador/a reaccione violenta- } \\
\text { mente cuando le hacen una falta }\end{array}$ & $66,7 \%$ & $22,2 \%$ & $11,1 \%$ & $0 \%$ & $76,9 \%$ & $19,2 \%$ & $3,8 \%$ & $0 \%$ \\
\hline $\begin{array}{l}\text { Ítem 9. Entrar duro está justificado si el público anima a } \\
\text { hacerlo }\end{array}$ & $50 \%$ & $23,1 \%$ & $15,4 \%$ & $11,5 \%$ & $69,2 \%$ & $19,2 \%$ & $3,8 \%$ & $7,7 \%$ \\
\hline $\begin{array}{l}\text { Ítem } 12 . \text { Está bien que un jugador/a proteste al árbitro sus } \\
\text { decisiones }\end{array}$ & $14,8 \%$ & $25,9 \%$ & $25,9 \%$ & $33,3 \%$ & $34,6 \%$ & $26,9 \%$ & $19,2 \%$ & $19,2 \%$ \\
\hline $\begin{array}{l}\text { Ítem } 15 . \text { Está bien que un entrenador/a anime a lesionar a } \\
\text { un jugador del equipo contrario }\end{array}$ & $81,5 \%$ & $11,1 \%$ & $3,7 \%$ & $3,7 \%$ & $88,5 \%$ & $11,5 \%$ & $0 \%$ & $0 \%$ \\
\hline $\begin{array}{l}\text { Ítem 17. Un contrario/a que está a punto de marcar gol tiene } \\
\text { que ser detenido/a de cualquier forma, legalmente o no }\end{array}$ & $44,4 \%$ & $25,9 \%$ & $14,8 \%$ & $14,8 \%$ & $76,9 \%$ & $11,5 \%$ & $0 \%$ & $11,5 \%$ \\
\hline $\begin{array}{l}\text { Ítem } 20 . \text { En los partidos importantes todo está permitido } \\
\text { para poder ganar }\end{array}$ & $48,1 \%$ & $37 \%$ & $3,7 \%$ & $11,1 \%$ & $85,5 \%$ & $3,8 \%$ & $7,7 \%$ & $0 \%$ \\
\hline $\begin{array}{l}\text { Ítem 22. Jugar duro se acepta si el otro equipo también } \\
\text { juega duro }\end{array}$ & $29,6 \%$ & $29,6 \%$ & $11,1 \%$ & $29,6 \%$ & $38,5 \%$ & $23,1 \%$ & $19,2 \%$ & $19,2 \%$ \\
\hline $\begin{array}{l}\text { Ítem 24. Un jugador/a hábil debe ser parado siempre de } \\
\text { forma limpia }\end{array}$ & $7,4 \%$ & $7,4 \%$ & $22,2 \%$ & $63 \%$ & $19,2 \%$ & $19,2 \%$ & $19,2 \%$ & $42,3 \%$ \\
\hline $\begin{array}{l}\text { Ítem 25. Los oponentes rápidos tienen que ser detenidos de } \\
\text { cualquier manera, legalmente o no }\end{array}$ & $37 \%$ & $44,4 \%$ & $7,4 \%$ & $11,1 \%$ & $61,5 \%$ & $15,4 \%$ & $15,4 \%$ & $7,7 \%$ \\
\hline $\begin{array}{l}\text { Ítem 26. El entrenador/a debería prohibir cometer entradas } \\
\text { duras en los partidos }\end{array}$ & $14,8 \%$ & $18,5 \%$ & $22,2 \%$ & $44,4 \%$ & $38,5 \%$ & $3,8 \%$ & $19,2 \%$ & $38,5 \%$ \\
\hline $\begin{array}{l}\text { Ítem 27. Está bien que un equipo intente molestar o enfa- } \\
\text { dar a un jugador/a impulsivo del equipo contrario }\end{array}$ & $70,4 \%$ & $18,5 \%$ & $3,7 \%$ & $7,4 \%$ & $73,1 \%$ & $19,2 \%$ & $0 \%$ & $7,7 \%$ \\
\hline
\end{tabular}

C) Resultados según la práctica de fútbol federado

En general, futbolistas y no futbolistas muestran actitudes bastante favorables al desarrollo del juego por los cauces de la deportividad, aunque, al realizar el análisis estadístico, encontramos que existían diferencias significativas $(\mathrm{p}<0.05)$ entre ambas categorías en el ítem 20. El alumnado con experiencia futbolística se mostró más de acuerdo que el alumnado sin ella en cuanto al "todo vale" para conseguir la victoria.
Además de esto, en algunos casos puntuales que les hemos presentado existen datos que llaman la atención, como el de "está bien que un entrenador/a anime a lesionar a un jugador/a del equipo contrario", donde se encuentra que mientras que la totalidad de los no futbolistas encuestados mostró su rotundo desacuerdo, un pequeño porcentaje de futbolistas admitió si estarlo, como vemos a continuación:

Tabla 6. Porcentajes de respuesta según la práctica fútbol federado para la categoría juego limpio.

\begin{tabular}{|c|c|c|c|c|c|c|c|c|}
\hline \multirow{2}{*}{ ¿En qué grado estás de acuerdo con que...? } & \multicolumn{4}{|c|}{ Futbolistas } & \multicolumn{4}{|c|}{ No futbolistas } \\
\hline & Nada & Poco & Bastante & Total & Nada & Poco & Bastante & Total \\
\hline $\begin{array}{l}\text { Ítem 6. Está bien que un jugador/a reaccione violenta- } \\
\text { mente cuando le hacen una falta }\end{array}$ & $70,6 \%$ & $17,6 \%$ & $11,8 \%$ & $0 \%$ & $72,2 \%$ & $22,2 \%$ & $5,6 \%$ & $0 \%$ \\
\hline $\begin{array}{l}\text { Ítem 9. Entrar duro está justificado si el público anima a } \\
\text { hacerlo }\end{array}$ & $56,3 \%$ & $25 \%$ & $18,8 \%$ & $0 \%$ & $61,1 \%$ & $19,4 \%$ & $13,9 \%$ & $5,6 \%$ \\
\hline $\begin{array}{l}\text { Ítem 12. Está bien que un jugador/a proteste al árbitro sus } \\
\text { decisiones }\end{array}$ & $17,6 \%$ & $23,5 \%$ & $17,6 \%$ & $41,2 \%$ & $27,8 \%$ & $27,8 \%$ & $25 \%$ & $19,4 \%$ \\
\hline $\begin{array}{l}\text { Ítem 15. Está bien que un entrenador/a anime a lesionar a } \\
\text { un jugador del equipo contrario }\end{array}$ & $76,5 \%$ & $11,8 \%$ & $5,9 \%$ & $5,9 \%$ & $88,9 \%$ & $11,1 \%$ & $0 \%$ & $0 \%$ \\
\hline $\begin{array}{l}\text { Ítem 17. Un contrario/a que está a punto de marcar gol tiene } \\
\text { que ser detenido/a de cualquier forma, legalmente o no }\end{array}$ & $47,1 \%$ & $17,6 \%$ & $23,5 \%$ & $11,8 \%$ & $66,7 \%$ & $19,4 \%$ & $0 \%$ & $13,9 \%$ \\
\hline $\begin{array}{l}\text { Îtem 20. En los partidos importantes todo está permitido } \\
\text { para poder ganar }\end{array}$ & $47,1 \%$ & $35,3 \%$ & $5,9 \%$ & $11,8 \%$ & $77,8 \%$ & $13,9 \%$ & $5,6 \%$ & $2,8 \%$ \\
\hline
\end{tabular}




\begin{tabular}{|c|c|c|c|c|c|c|c|c|}
\hline \multirow{2}{*}{ ¿En qué grado estás de acuerdo con que...?? } & \multicolumn{4}{|c|}{ Futbolistas } & \multicolumn{4}{|c|}{ No futbolistas } \\
\hline & Nada & Poco & Bastante & Total & Nada & Poco & Bastante & Total \\
\hline $\begin{array}{l}\text { Ítem 22. Jugar duro se acepta si el otro equipo también } \\
\text { juega duro }\end{array}$ & $23,5 \%$ & $41,2 \%$ & $5,9 \%$ & $29,4 \%$ & $39,9 \%$ & $19,4 \%$ & $19,4 \%$ & $22,2 \%$ \\
\hline $\begin{array}{l}\text { Ítem } 24 . \text { Un jugador/a hábil debe ser parado siempre de } \\
\text { forma limpia }\end{array}$ & $5,9 \%$ & $5,9 \%$ & $35 \%$ & $52,9 \%$ & $16,7 \%$ & $16,7 \%$ & $13,9 \%$ & $52,8 \%$ \\
\hline $\begin{array}{l}\text { Ítem } 25 . \text { Los oponentes rápidos tiene que ser detenidos de } \\
\text { cualquier manera, legalmente o no }\end{array}$ & $35,3 \%$ & $47,1 \%$ & $5,9 \%$ & $11,8 \%$ & $55,6 \%$ & $22,2 \%$ & $13,9 \%$ & $8,3 \%$ \\
\hline $\begin{array}{l}\text { Ítem 26. El entrenador/a debería prohibir cometer entradas } \\
\text { duras en los partidos }\end{array}$ & $5,9 \%$ & $17,6 \%$ & $35,3 \%$ & $41,2 \%$ & $36,1 \%$ & $8,3 \%$ & $13,9 \%$ & $41,7 \%$ \\
\hline $\begin{array}{l}\text { Ítem } 27 . \text { Está bien que un equipo intente molestar o enfa- } \\
\text { dar a un jugador/a impulsivo del equipo contrario }\end{array}$ & $64,7 \%$ & $23,5 \%$ & $5,9 \%$ & $5,9 \%$ & $75 \%$ & $16,7 \%$ & $8,3 \%$ & $0 \%$ \\
\hline
\end{tabular}

También llama la atención el caso de la protesta arbitral, en el que encontramos que un $58 \%$ de los futbolistas frente a un $44 \%$ de los no futbolistas, se muestra favorable a las quejas.

\section{Resultados sobre la victoria}

\section{A) Resultados totales}

En lo referente a las preguntas sobre la importancia de la victoria, las actitudes del alumnado a favor y en contra de dar más importancia a la victoria que a los medios utilizados para su obtención, están muy igualadas, prueba de ello es la respuesta al primer ítem (7) referente a la victoria, donde observamos que la mitad del alumnado encuestado considera que la victoria sí es lo más importante, y la otra mitad considera que no lo es. Mediante estas respuestas podemos observar que, aunque la victoria es un aspecto bastante importante para ellos y ellas en el juego, no consideran que cualquier medio sea lícito para ganar, como vemos en sus respuestas a preguntas como "Está bien que un jugador logre un penalti dejándose caer dentro del área" (De acuerdo 13,2\%, desacuerdo $86,8 \%$ ). Sin embargo, observamos que sí pueden llegar a defender otras estrategias consideradas más "morales" para ganar, como perder tiempo para asegurar un resultado favorable (36\% de acuerdo):

Tabla 7. Porcentajes generales de respuesta para la categoría victoria.

\begin{tabular}{lcccc}
\hline ¿En qué grado estás de acuerdo con que...? & Nada & Poco & Bastante & Totalmente \\
\hline Ítem 7. En el fútbol, el resultado final es lo más importante & $11,5 \%$ & $38,5 \%$ & $25 \%$ & $25 \%$ \\
\hline Ítem 10. En un partido el principal objetivo es conseguir la victoria & $15,1 \%$ & $41,1 \%$ & $15,1 \%$ & $28,3 \%$ \\
\hline Ítem 13. Lo más importante en un campeonato es ser el primero en la clasificación & $21,2 \%$ & $36,5 \%$ & $17,3 \%$ & $25 \%$ \\
\hline Ítem 16. Lo más importante en el fútbol es marcar goles, da igual cómo se hagan & $28,3 \%$ & $39,6 \%$ & $11,3 \%$ & $20,8 \%$ \\
\hline Ítem 18. Está bien que un equipo pierda tiempo si va ganando para asegurar la victoria & $44,2 \%$ & $19,2 \%$ & $21,2 \%$ & $15,4 \%$ \\
\hline Ítem 21. Está bien que un jugador/a logre un penalti, dejándose caer dentro del área & $60,4 \%$ & $26,4 \%$ & $5,7 \%$ & $7,5 \%$ \\
\hline Ítem 23. En el fútbol es más importante ganar que divertirse & $39,6 \%$ & $28,3 \%$ & $7,5 \%$ & $24,5 \%$ \\
\hline
\end{tabular}

A través del análisis de los grupos de discusión, se ha podido corroborar esta teoría: Cuando se les presentan situaciones en las que tienen que decantarse por la diversión o por la victoria, encontramos que, generalmente, los encuestados eligen la victoria si lo que está en juego es algo importante, como quedar primero en un campeonato, aunque matizan que, si la victoria se consigue de forma injusta, como por ejemplo con un penalti que no era, la satisfacción no es la misma. La opinión cambia si la victoria se consigue con un gol en propia puerta del equipo contrario, donde parece ser que si se encuentran satisfechos dado que no asocian la ventaja a una injusticia si no a un error del contrario:

B) Resultados según sexo 
Tabla 8. Porcentajes de respuesta según sexo para la categoría victoria.

\begin{tabular}{|c|c|c|c|c|c|c|c|c|}
\hline \multirow[t]{2}{*}{ ¿En qué grado estás de acuerdo con que...? } & \multicolumn{4}{|c|}{ Chicos } & \multicolumn{4}{|c|}{ Chicas } \\
\hline & Nada & Poco & Bastante & Total & Nada & Poco & Bastante & Total \\
\hline Ítem 7. En el fútbol el resultado final es lo más importante & $3,7 \%$ & $25,9 \%$ & $25,9 \%$ & $44,4 \%$ & $20 \%$ & $52 \%$ & $24 \%$ & $4 \%$ \\
\hline $\begin{array}{l}\text { Ítem 10. En un partido el principal objetivo es conseguir la } \\
\text { victoria }\end{array}$ & $3,7 \%$ & $29,6 \%$ & $18,5 \%$ & $48,1 \%$ & $26,9 \%$ & $53,8 \%$ & $11,5 \%$ & $7,7 \%$ \\
\hline $\begin{array}{l}\text { Ítem 13. Lo más importante en un campeonato es ser el } \\
\text { primero en la clasificación }\end{array}$ & $11,1 \%$ & $29,6 \%$ & $14,8 \%$ & $44,4 \%$ & $32 \%$ & $44 \%$ & $20 \%$ & $4 \%$ \\
\hline $\begin{array}{l}\text { Ítem 16. Lo más importante en el fútbol es marcar goles, da } \\
\text { igual cómo se marquen }\end{array}$ & $22,2 \%$ & $37 \%$ & $14,8 \%$ & $25,9 \%$ & $34,6 \%$ & $42,3 \%$ & $7,7 \%$ & $15,4 \%$ \\
\hline $\begin{array}{l}\text { Ítem } 18 \text {. Está bien que un equipo pierda tiempo si va ga- } \\
\text { nando para asegurar la victoria }\end{array}$ & $29,6 \%$ & $14,8 \%$ & $33,3 \%$ & $22,2 \%$ & $60 \%$ & $24 \%$ & $8 \%$ & $8 \%$ \\
\hline $\begin{array}{l}\text { Ítem 21. Está bien que un jugador/a logre un penalti, deján- } \\
\text { dose caer dentro del área }\end{array}$ & $51,9 \%$ & $25,9 \%$ & $11,1 \%$ & $11,1 \%$ & $69,2 \%$ & $26,9 \%$ & $0 \%$ & $3,8 \%$ \\
\hline Ítem 23. En el fútbol es más importante ganar que divertirse & $22,2 \%$ & $29.6 \%$ & $11,1 \%$ & $37 \%$ & $57,7 \%$ & $26,9 \%$ & $3,8 \%$ & $11,5 \%$ \\
\hline
\end{tabular}

Los resultados de la prueba U de Mann Whitney, determinaron que existen diferencias significativas $(\mathrm{p}<0.05)$ entre chicos y chicas en ciertas actitudes hacia la consecución de la victoria en el juego. La mayoría de los chicos, a diferencia de las chicas, se muestran resultadistas en lo que al fútbol se refiere, esto es, opinan cosas como que en el fútbol, el resultado final es lo más importante (Ítem 7, 70,4 \% chicos, $28 \%$ chicas), que en un partido el principal objetivo es conseguir la victoria (ítem 10, 66,7 \% de chicos frente a sólo el 19,2 \% de las chicas), o que lo más importante en un campeonato es ser el primero en la clasificación (ítem 13, 59,3 \% Vs. 24 \%). Además, a más de la mitad de los chicos encuestados no le importaría conseguir la victoria mediante medios poco éticos como perder tiempo (55,6 \% Vs. $16 \%)$. Destaca también la diferencia entre chicos y chicas frente a lo piensan que es más importante en el fútbol: mientras que el $48 \%$ de los chicos defiende que ganar es más importante que divertirse, sólo el 15,4 \% de las chicas antepondría la victoria a la satisfacción personal y el disfrute.
A pesar de que estadísticamente no existen diferencias significativas entre chicos y chicas en las respuestas al ítem 21, llama la atención que un porcentaje más que considerable de ellos (22,2\%) simularía un penalti con tal de ganar.

Viendo estos datos, y de acuerdo también a lo analizado mediante el grupo de discusión, se puede afirmar que los chicos muestran un mayor afán por conseguir la victoria, respecto a las chicas, que anteponen la diversión y el disfrute en la práctica a la consecución del objetivo en sí.

Como se ha comentado en los resultados generales, aunque los chicos muestran mayor competitividad y le dan una gran importancia a ganar, la mayoría no se encuentra conforme con la victoria cuando ésta se consigue con medios injustos (como ellos mismos califican en la entrevista del grupo de discusión) por lo que no les aporta la misma satisfacción y disfrute.

C) Resultados según la práctica de fútbol federado

Tabla 9. Porcentajes de respuesta según práctica fútbol federado para la categoría victoria

\begin{tabular}{|c|c|c|c|c|c|c|c|c|}
\hline \multirow[t]{2}{*}{ ¿En qué grado estás de acuerdo con que...? } & \multicolumn{4}{|c|}{ Futbolistas } & \multicolumn{4}{|c|}{ No futbolistas } \\
\hline & Nada & Poco & Bastante & Total & Nada & Poco & Bastante & Total \\
\hline Ítem 7. En el fútbol el resultado final es lo más importante & $0 \%$ & $23,5 \%$ & $35,3 \%$ & $41,2 \%$ & $17,1 \%$ & $45,7 \%$ & $20 \%$ & $17,1 \%$ \\
\hline $\begin{array}{l}\text { Ítem 10. En un partido el principal objetivo es conseguir la } \\
\text { victoria }\end{array}$ & $0 \%$ & $17,6 \%$ & $23,5 \%$ & $58,8 \%$ & $22,2 \%$ & $52,8 \%$ & $11,1 \%$ & $13,9 \%$ \\
\hline $\begin{array}{l}\text { Ítem 13. Lo más importante en un campeonato es ser el } \\
\text { primero en la clasificación }\end{array}$ & $0 \%$ & $23,5 \%$ & $23,5 \%$ & $52,9 \%$ & $31,4 \%$ & $42,9 \%$ & $14,3 \%$ & $11,4 \%$ \\
\hline $\begin{array}{l}\text { Ítem 16. Lo más importante en el fútbol es marcar goles, da } \\
\text { igual cómo se marquen }\end{array}$ & $17,6 \%$ & $35,3 \%$ & $11,8 \%$ & $38,3 \%$ & $33,3 \%$ & $41,7 \%$ & $11,1 \%$ & $13,9 \%$ \\
\hline $\begin{array}{l}\text { Ítem 18. Está bien que un equipo pierda tiempo si va ga- } \\
\text { nando para asegurar la victoria }\end{array}$ & $54,3 \%$ & $20 \%$ & $17,1 \%$ & $8,6 \%$ & $23,5 \%$ & $17,6 \%$ & $29,4 \%$ & $29,4 \%$ \\
\hline $\begin{array}{l}\text { Ítem 21. Está bien que un jugador/a logre un penalti, deján- } \\
\text { dose caer en el área }\end{array}$ & $47,1 \%$ & $29,4 \%$ & $17,6 \%$ & $5,9 \%$ & $66,7 \%$ & $25 \%$ & $0 \%$ & $8,3 \%$ \\
\hline Ítem 23. En el fútbol es más importante ganar que divertirse & $17,6 \%$ & $35,3 \%$ & $11,8 \%$ & $35,3 \%$ & $50 \%$ & $25 \%$ & $5,6 \%$ & $19,4 \%$ \\
\hline
\end{tabular}


Como los resultados de la tabla 9 muestran y de acuerdo al análisis mediante la U de Mann Whitney, hubo diferencias significativas entre alumnado futbolista y no futbolista $(\mathrm{p}<$ 0.05 ) en lo que a la importancia de la victoria en el fútbol se refiere, concretamente, y de nuevo, en lo relativo a los ítems 7 , $10,13,18$ y 23. La mayor parte de los futbolistas encuestados sitúan a la victoria como el objetivo principal en el fútbol, y aunque afirman que para conseguirla no es lícito violar las reglas constitutivas del fútbol (las que están escritas), si ven con buenos ojos utilizar conductas contrarias al reglamento, pero legitimizadas por la práctica, como perder tiempo cuando se va ganando (58,8 \% de los futbolistas de acuerdo): La mayoría de chicos que dijeron ser futbolistas federados (en esta muestra no existía ninguna chica que jugara al fútbol), defendieron los resultados por encima de otros aspectos, afirmando que en el fútbol, el resultado final es lo más importante $(76,4 \%)$, contrarios a la opinión de la mayoría de alumnado no practicante $(62,9 \%)$, que el principal objetivo es la victoria ( $82 \%$ frente al $25 \%$ o que ser el primero en la clasificación es lo importante $(76,5 \%$, casi el mismo porcentaje de los no futbolistas que opinan todo lo contrario: $74,3 \%$ ). Una vez más, las diferencias son considerablemente grandes entre futbolistas y no futbolistas, en lo que a la importancia de la victoria se refiere.

Con estos datos y los del grupo de discusión, se observa que existen diferencias significativas entre las opiniones de futbolistas y no futbolistas en este ámbito de la investigación, para ello basta con observar algunas de sus respuestas cuando se les presentaron dos situaciones en las que tenían que elegir qué final preferían: en la primera se les propuso jugar bien un partido y perderlo en una jugada desafortunada al final, y en la segunda lo contrario; jugar mal y ganar de penalti dudoso en los últimos minutos:

\section{Grupo de discusión-2}

Moderadora: Entonces tú, ¿cuál preferirías?

Raúl*: La segunda.

Moderadora: Ganar de penalti injusto.

Raúl*: Sí.

Raúl*: Bueno, mientras que ganes...

Moderadora: Tú Karolina.

Karolina: Yo la primera.

Moderadora: Tú preferirías divertirte, aunque luego perdieses.

Karolina: Si, porque en la primera se merecía ganar el otro equipo porque es superior.

Moderadora: Pero, ¿si te estás jugando algo preferirías ganar?

Karolina: No, porque sería injusto.

* Se ha señalado con un asterisco el alumno que, en este caso, era jugador de fútbol federado.

\section{Resultados sobre la igualdad de género}

A) Resultados generales:

Tabla 10. Porcentajes de respuesta generales para la categoría igualdad de género.

\begin{tabular}{lcccc}
\hline ¿En qué grado estás de acuerdo con que...? & Nada & Poco & Bastante & Totalmente \\
\hline Ítem 8. El fútbol es un deporte más apropiado para chicos que para chicas & $65,4 \%$ & $15,4 \%$ & $11,5 \%$ & $7,7 \%$ \\
\hline Ítem 11. A las chicas les puede gustar el fútbol & $0 \%$ & $7,7 \%$ & $13,5 \%$ & $78,8 \%$ \\
\hline Ítem 14. Las competiciones femeninas de fútbol son poco emocionantes & $58.5 \%$ & $28,3 \%$ & $5,7 \%$ & $7,5 \%$ \\
\hline Ítem 19. Las chicas pueden jugar al fútbol igual de bien que los chicos & $0 \%$ & $7,5 \%$ & $17 \%$ & $75,5 \%$ \\
\hline Ítem 28. Las chicas que practican fútbol suelen ser poco femeninas & $54,7 \%$ & $20,8 \%$ & $17 \%$ & $7,5 \%$ \\
\hline Ítem 29. Los partidos de fútbol femenino pueden ser bonitos & $13,2 \%$ & $9,4 \%$ & $20,8 \%$ & $58,6 \%$ \\
\hline
\end{tabular}

Por lo que respecta a las preguntas referentes a la igualdad entre hombres y mujeres en el ámbito futbolístico, en general, existe una actitud de acuerdo hacia la figura de la mujer en el fútbol, es decir, la mayoría ven normal que las mujeres quieran y puedan jugar al fútbol, o que puedan ser mejores en ello que algunos hombres y aceptan que las competiciones femeninas pueden ser entretenidas, aunque es relevante, por ejemplo, que aún un alto porcentaje de los jóvenes encuestados creen que el fútbol es un deporte más adecuado para chicos que para chicas (19\%), dudan de que un partido de fútbol femenino pueda ser bonito (33\%) o, en cuanto a la estética de las mujeres que juegan al fútbol, prácticamente una cuarta parte de los encuestados asegura que las chicas futbolistas son poco femeninas, con lo cual y tal y como se deja entrever en el grupo de discusión, parece ser que todavía continúan algunos viejos prejuicios en este sentido:

\section{Grupo de discusión-1}

Moderadora: Pero, ¿̇me las podéis describir físicamente?, si son gordas, flacas, altas, bajas... (En referencia a una imagen de dos chicas jugando al fútbol)

Bianka: Yo no digo que no sean cuidadosas, pero que no son “¡ay! me he roto una uña”. 


\section{Grupo de discusión-2}

Karolina: Están fuertes

Roy: Tienen todas una coleta.

También llama la atención, a pesar de que la mayoría están de acuerdo en que la mujer practique fútbol, el desconocimiento de la situación del fútbol femenino, ya que en los grupos de discusión, chicos y chicas afirmaron no saber que competiciones femeninas se juegan, incluso algunos dijeron no haber visto nunca jugar al fútbol a una mujer. Esto es una prueba de la poca visibilidad que tiene el fútbol femenino en nuestro país, eclipsado totalmente por la práctica de los chicos, lo que no hace más que seguir asociándolo al carácter masculino.

B) Resultados según sexo:

Se aprecian diferencias significativas $(\mathrm{p}<0.05)$ entre lo que piensan chicos y chicas acerca de que la mujer practique fút- bol (ítems 8 y 14). Aunque la mayoría desmiente que el fútbol no es un deporte más apropiado para hombres que para mujeres, un porcentaje más que significativo de los chicos $(33,3$ $\%$ según vemos en la tabla 11) si se mostró de acuerdo con esta idea. Además, casi un $15 \%$ de ellos frente a un $10 \%$ de chicas, dijo que los partidos de fútbol femenino carecen de emoción.

También se encuentran diferencias importantes en lo referente a cómo perciben estéticamente a las futbolistas y al fútbol femenino. El porcentaje de chicas que piensan que las jugadoras de fútbol no son femeninas, es mayor que el de los chicos que piensan lo mismo (30,7 \% frente al 18,5\%), al igual que en la afirmación "los partidos de fútbol femenino pueden ser bonitos", en el que son más las chicas que no están de acuerdo que los chicos, con lo cual podemos comprobar que todavía hoy en día existen muchos tabúes relacionados con la mujer y alimentados, precisamente, por ellas mismas:

Tabla 11. Porcentajes de respuesta según sexo para la categoría igualdad de género.

\begin{tabular}{|c|c|c|c|c|c|c|c|c|}
\hline \multirow{2}{*}{$\begin{array}{l}\text { ¿En qué grado estás de acuerdo con } \\
\text { que...? }\end{array}$} & \multicolumn{4}{|l|}{ Chicos } & \multicolumn{4}{|l|}{ Chicas } \\
\hline & Nada & Poco & Bastante & Total & Nada & Poco & Bastante & Total \\
\hline $\begin{array}{l}\text { Ítem } 8 . \text { El fútbol es un deporte más } \\
\text { apropiado para chicos que para chicas }\end{array}$ & $55,6 \%$ & $11,1 \%$ & $22,2 \%$ & $11,1 \%$ & $76 \%$ & $20 \%$ & $0 \%$ & $4 \%$ \\
\hline $\begin{array}{l}\text { Ítem 11. A las chicas les puede gustar } \\
\text { el fútbol }\end{array}$ & $0 \%$ & $7,7 \%$ & $23,1 \%$ & $69,2 \%$ & $0 \%$ & $7,7 \%$ & $3,8 \%$ & $88,5 \%$ \\
\hline $\begin{array}{l}\text { Ítem 14. Las competiciones femeninas } \\
\text { de fútbol son poco emocionantes }\end{array}$ & $40,7 \%$ & $44,4 \%$ & $3,7 \%$ & $11,1 \%$ & $76,9 \%$ & $11,5 \%$ & $7,7 \%$ & $3,8 \%$ \\
\hline $\begin{array}{l}\text { Ítem 19. Las chicas pueden jugar al fút- } \\
\text { bol igual de bien que los chicos }\end{array}$ & $0 \%$ & $11,1 \%$ & $22,2 \%$ & $66,7 \%$ & $0 \%$ & $3,8 \%$ & $11,5 \%$ & $84,6 \%$ \\
\hline $\begin{array}{l}\text { Ítem 28. Las chicas que practican } \\
\text { fútbol suelen ser poco femeninas }\end{array}$ & $59,3 \%$ & $22,2 \%$ & $14,8 \%$ & $3,7 \%$ & $50 \%$ & $19,2 \%$ & $19,2 \%$ & $11,5 \%$ \\
\hline $\begin{array}{l}\text { Ítem 29. Los partidos de fútbol fe- } \\
\text { menino pueden ser bonitos }\end{array}$ & $14,8 \%$ & $0 \%$ & $33,3 \%$ & $51,9 \%$ & $11,5 \%$ & $19,2 \%$ & $7,7 \%$ & $61,5 \%$ \\
\hline
\end{tabular}

Como se ve, todavía un buen porcentaje de chicos se muestra escéptico ante el hecho de que las mujeres practiquen fútbol. Así lo cuentan ellos y ellas en las entrevistas del grupo de discusión:

\section{Grupo de discusión-2}

Moderadora: ¿Pensáis que el fútbol es un deporte para chicos?

Ainhoa: Yo lo que creo es que normalmente los chicos son más brutos, y aquí en España es más interesante un partido de chicos que de chicas, porque aquí no se ve el femenino.

David: ... para mí no es interesante, no hay tanto cuerpo...
Moderadora: ¿Por qué no salen en la tele Roy?

Roy: Porque los chicos, y yo también me incluyo, hemos ocupado como todos los puestos de la tele, entonces...

\section{Grupo de discusión-1}

Adrián: (Habla de por qué no se ve fútbol femenino en la televisión) Pero yo creo que por dos cosas: porque el fútbol masculino lleva toda la vida, desde siempre, el fútbol femenino es algo que ahora está empezando a conocer la gente...

Moderadora: ¿Tú crees? Lleva muchos años el fútbol femenino.

Elsa: Es machismo. 
C) Resultados según la práctica de fútbol federado

Existen diferencias de respuesta significativas $(p<0.05)$ entre el alumnado futbolista y no futbolista en cuanto a la aceptación de la participación de la mujer en el fútbol, concretamente en lo referido al fútbol considerado como un deporte de chicos (ítem 8). Aunque en general todos los encuestados y encuestadas están en desacuerdo ante la afirmación de que el fútbol es un deporte más apropiado para chicos que para chicas, un porcentaje cercano a la mitad de futbolistas $(41,2$ $\%)$ se muestra de acuerdo.

También llaman la atención algunos datos que se des- prenden de las respuestas del cuestionario: los futbolistas si piensan que un partido de fútbol femenino puede ser bonito, y sólo el $5 \%$ de éstos opina lo contrario, y curiosamente el $30,6 \%$ de los chicos y chicas que no eran futbolistas no creen que un partido entre dos equipos de chicas sea vistoso. Además, ningún futbolista se mostró reacio a la idea de que a las chicas les pueda gustar el fútbol, y si un $11 \%$ de los no futbolistas. En cuanto a la estética de las chicas futbolistas, aunque la mayoría tiene opiniones positivas, llama la atención que un porcentaje considerable, tanto de futbolistas como de no futbolistas, considere que son poco femeninas (17\% y $27 \%$, respectivamente).

Tabla 12. Porcentajes de respuesta según práctica de fútbol federado para la categoría igualdad de género.

\begin{tabular}{|c|c|c|c|c|c|c|c|c|}
\hline \multirow[t]{2}{*}{ ¿En qué grado estás de acuerdo con que...? } & \multicolumn{4}{|c|}{ Futbolistas } & \multicolumn{4}{|c|}{ No futbolistas } \\
\hline & Nada & Poco & Bastante & Total & Nada & Poco & Bastante & Total \\
\hline $\begin{array}{l}\text { Ítem } 8 . \text { El fútbol es un deporte más apropiado para chicos } \\
\text { que para chicas }\end{array}$ & $47,1 \%$ & $11,8 \%$ & $29,4 \%$ & $11,8 \%$ & $74,3 \%$ & $17,1 \%$ & $2,9 \%$ & $5,7 \%$ \\
\hline Ítem 11. A las chicas les puede gustar el fútbol & $0 \%$ & $0 \%$ & $37,5 \%$ & $62,5 \%$ & $0 \%$ & $11,1 \%$ & $2,8 \%$ & $86,1 \%$ \\
\hline $\begin{array}{l}\text { Ítem 14. Las competiciones femeninas de fútbol son poco } \\
\text { emocionantes }\end{array}$ & $41,2 \%$ & $47,1 \%$ & $0 \%$ & $11,8 \%$ & $66,7 \%$ & $19,4 \%$ & $8,3 \%$ & $5,6 \%$ \\
\hline $\begin{array}{l}\text { Ítem 19. Las chicas pueden jugar al fútbol igual de bien que } \\
\text { los chicos }\end{array}$ & $0 \%$ & $11,8 \%$ & $17,6 \%$ & $70,6 \%$ & $5,6 \%$ & $0 \%$ & $16,7 \%$ & $77,8 \%$ \\
\hline $\begin{array}{l}\text { Ítem 28. Las chicas que practican fútbol suelen ser poco } \\
\text { femeninas }\end{array}$ & $64,7 \%$ & $17,6 \%$ & $17,6 \%$ & $0 \%$ & $50 \%$ & $22,2 \%$ & $16,7 \%$ & $11,1 \%$ \\
\hline Ítem 29.Los partidos de fútbol femenino pueden ser bonitos & $5,9 \%$ & $0 \%$ & $35,3 \%$ & $58,8 \%$ & $16,7 \%$ & $13,9 \%$ & $13,9 \%$ & $55,6 \%$ \\
\hline
\end{tabular}

\section{Discusión}

\section{A) Para la categoría catalogada como juego limpio}

Tomando como referencia la variable sexo, se observan pequeñas diferencias significativas entre chicos y chicas, lo cual quiere decir que el sexo es determinante en ciertas actitudes hacia jugar limpio en el fútbol. Así se observa también en el estudio de Gómez Mármol et al. (2011, p.40), que muestra que los hombres, en este caso tenistas y futbolistas, tienen "una menor aproximación a la deportividad en todas sus dimensiones”. En el caso que nos ocupa, esta diferencia entre niños y niñas puede ser debida a que, probablemente, ellos consumen más fútbol que ellas, y, por ende, tienden a defender y reflejar las actitudes que pueden ver en el fútbol por parte del público.En términos generales, todos se muestran favorables a la deportividad en el juego, aunque como hemos comentado en el análisis, se vuelven más tolerantes hacia el juego sucio si el otro equipo también lo hace. Estos resultados contradicen una de las conclusiones del estudio que Velázquez y Hernández (2002), cuando afirman que el alumnado se muestra más favorable a responder con juego limpio, por ejemplo, dialogando con el rival o con el árbitro, al juego duro del equipo oponente.
También se observan algunas diferencias significativas entre futbolistas y no futbolistas en lo relativo al fair play, donde todos y todas se muestran bastante favorables al respeto por las normas, aunque sí es cierto que se ha podido comprobar que los futbolistas muestran actitudes sensiblemente mayores a favor de jugar duro en el desarrollo de un partido. Se podría suponer que estas actitudes vienen condicionadas por el discurso de muchos entrenadores y entrenadoras que, como contaban en los grupos de discusión, animan a los jugadores/ as a no evitar el contacto físico en los lances del juego para conseguir los objetivos.

Estos resultados se encuentran en la misma línea que los que muestran algunos estudios previos sobre fair play y deporte, como el de Cruz et al. (1991), donde destacan que uno de los valores frecuentes mostrados por los futbolistas de su estudio era la utilidad como parte del equipo por medio de faltas. Teniendo en cuenta, que aunque las diferencias entre no futbolistas y futbolistas no son demasiado significativas pero que éstos últimos son más permisivos al juego duro, en cierto modo, también se puede considerar que los resultados obtenidos concuerdan con los de estudios como los de Cecchini, González y Montero (2007) que dice que la práctica de deportes de contacto medio, como el fútbol o el baloncesto, predicen bajos resultados de fair play o Cruz, Capdevila, 
Boixadós, Pintanel, Alonso, Mimbrero y Torregrosa (1996), en el que afirman que los niños futbolistas encuestados tienen una actitud de indiferencia hacia el juego duro, es decir, ni se terminan de inclinar a las actitudes anti deportivas ni las terminan de rechazar.

En síntesis, se ha podido comprobar que el juego limpio está condicionado por el sexo o por la práctica de fútbol federado. Los niños y los futbolistas muestran actitudes sensiblemente mayores hacia jugar duro que las niñas o los no futbolistas, lo cual puede estar condicionado por la experiencia en la práctica de esta población, contaminada por la exigencia de entrenadores y de la competición.

\section{B) Discusión para la categoría catalogada como afán por la victoria}

Se concluye que las variables sexo y práctica de fútbol federado influyen sobre las actitudes hacia la consecución de la victoria.

Por un lado, los chicos muestran un mayor afán por conseguir éxito. Esto puede estar condicionado por los estereotipos sexuales a causa de las influencias socioculturales que les rodean, de los cuales los niños y niñas son agentes transmisores, por ejemplo: la distribución de papeles en el ámbito familiar, donde a los niños se les presupone competitividad, actividad, fuerza..., o los juegos y juguetes que reflejan un estereotipo social sexista, y fomentan las practicas competitivas, como los balones, pistolas o videojuegos. Esta diferencia de opiniones entre chicos y chicas acerca de la importancia de la victoria frente a otro tipo de logros también puede estar condicionada, en este estudio, por la práctica. Es decir, puesto que ninguna chica en este estudio jugaba al fútbol, es posible que su visión sobre lo que es más importante en el fútbol esté idealizada y/o no contaminada por la práctica y la competición en un equipo federado.

Por el otro, la práctica de fútbol federado también es determinante en cuanto a la importancia que se otorga a la victoria, siendo los chicos que practican fútbol federado los que presentan un mayor afán por ella. Esto posiblemente pueda deberse, a la influencia de los entrenadores o entrenadoras, que aunque se centren en enseñar a los niños y niñas los procedimientos del fútbol, es posible que se dejen contaminar por la competición y también les exijan resultados. Estos resultados contradicen los obtenidos por el equipo del GEPE en el trabajo que Cruz, Boixadós, Capdevila, Mimbrero, Torregrosa, y Valiente (1999)realizaron con estudiantes de la E.S.O. los cuales también concordaban con los de futbolistas jóvenes británicos en los que el valor ganar ocupaba las últimas posiciones. No obstante, estos resultados vienen a corroborar los de De Marziani (2008), en el que a partir de los datos obtenidos por un estudio publicado en "El País", se afirma que la mayoría de los practicantes de fútbol están presionados por las exigencias de conseguir éxito en este deporte y, consecuentemente, una amplia mayoría acaba abandonándolo.

\section{C) Discusión para la categoría catalogada como igualdad de género}

La imagen y/o opinión que se tiene de la mujer practicante de fútbol varía según el sexo o la práctica del fútbol por parte de los encuestados. En general, la tónica del análisis es que todos muestran una actitud bastante favorable a que la mujer practique fútbol, si bien es cierto, que los chicos y los futbolistas se muestran más escépticos en sus opiniones. Esto podría deberse a razones culturales, dado el contexto donde está realizado este estudio (España) donde el fútbol femenino todavía no tiene demasiada visibilidad y su práctica y seguimiento están muy masculinizados todavía. Esto viene a reforzar la antigua teoría de Snyder y Spreitzer (1983), en la que afirmaban que, aunque la actitud va variando con el paso de los ańos, el público tiene sus reservas en cuanto a la participación de la mujer en deportes culturalmente masculinos o de contacto, o la teoría de Postow (1980) que dice que el deporte del fútbol se considera masculino porque viene a reforzar un sentimiento de identidad de los hombres separados de las mujeres.

\section{Conclusiones}

Una vez realizada la discusión de los datos obtenidos y, de acuerdo con losobjetivos marcados para esta investigación, para la muestra de escolares con la que hemos trabajado, se ha concluido que, de forma general:

Para el objetivo "Conocer la visión que el alumnado tiene del fair play en el fútbol":

$1^{\circ}$. Existe una actitud favorable a jugar limpio en el fútbol, especialmente cuando se habla de contacto físico o reacciones violentas, no tanto así en lo referido a las protestas arbitrales.

$2^{\circ}$. Existen diferencias estadísticamente significativas entre chicos y chicas en relación al juego limpio en el fútbol, mostrando éstos, en general, actitudes menos favorables al fair play o juego limpio.

Para el objetivo "Conocer la importancia que el alumnado otorga a la victoria en el fútbol y a los fines para conseguirla":

$3^{\circ}$. Las actitudes de afán e indiferencia por la victoria están igualadas, siendo, según las respuestas, la victoria lo principal para la mitad del alumnado encuestado. $\mathrm{Si}$ bien, es cierto que no consideran lícitos todos los medios para conseguir el éxito.

$4^{\circ}$. La relación existente entre el sexo de los encuestados y su afán por conseguir la victoria sí muestra diferencias 
estadísticamente significativas, siendo los chicos los que demuestran actitudes más ambiciosas por el éxito y la victoria y las chicas por el disfrute.

Para el objetivo "Conocer la opinión que el alumnado tiene en cuanto a la participación de la mujer en el fútbol":

$5^{\circ}$. El alumnado muestra actitudes favorables en lo que a la participación de la mujer en el fútbol respecta, esto es, la mayoría acepta que las mujeres quiera y puedan jugar al fútbol y que destaquen en ello más que los hombres en ocasiones. No obstante, prácticamente un cuarto de la muestra mantiene la idea de que las mujeres futbolistas son poco femeninas.

$6^{\circ}$. La influencia de la variable sexo muestra valores significativos en relación con la igualdad de género en el fútbol. Los chicos se muestran más en desacuerdo en cuanto a la participación de la mujer en el fútbol y mantienen la idea de que el fútbol es un deporte más apropiado para hombres. No obstante, cabe destacar la percepción negativa que tienen un buen porcentaje de las chicas encuestadas acerca de la estética del fútbol femenino y las futbolistas.

Para el objetivo "Comprobar si la práctica de fútbol federado en el alumnado influye en la visión que tienen sobre el fair play, el papel de la mujer en el fútbol o la importancia que se le otorga a la victoria":

70. Se encuentran diferencias significativas entre futbolistas y no futbolistas en relación con el juego limpio. El alumnado futbolista muestra actitudes de tolerancia sensiblemente mayores al juego sucio en determinados casos.

$8^{\circ}$. La influencia de la práctica del fútbol sobre el afán de conseguir éxito o victoria, muestra valores estadísticamente significativos, esto es, el alumnado futbolista encuestado demuestra una mayor ambición por conseguir la victoria.

9. La práctica de fútbol federado ejerce influencia sobre la opinión que el alumnado tiene hacia la figura de la mujer en el fútbol. El alumnado futbolista muestra opiniones más reservadas en cuanto a la participación de la mujer en el fútbol en ciertos casos.

\section{Referencias}

1. Beregüí Gil, R., y Garcés de Los Fayos, E. J. (2007). Valores en el deporte escolar: estudio con profesores de educación física. Cuadernos de psicología del deporte, 7(2), 89-103.

2. Bisquerra, R. (2004). Metodología de la investigación educativa. Madrid: La Muralla.

3. Cruz, J., Boixadós, M., Valiente, L., Ruíz, A., Arbona, P., Molons, Z., Call, J., Berbel, G. y Capdevilla, L. (1991). Identificación de valores relevantes en jugadores jóvenes de fútbol. Revista de Investigación y Documentación sobre las Ciencias de la Educación Física y del Deporte, 19, 81-99.

4. Cruz, J., Boixadós, M., Torregrosa M., y Mimbrero J. (1996) ¿Existe un deporte educativo?: Papel de las competiciones deportivas en el proceso de socialización del niño. Revista de psicología del deporte. 9-10

5. Cruz, J., Capdevila, L., Boixadós, M., Pintanel, M., Alonso, C., Mimbrero, J., y Torregrosa, M. (1996). Identificación de conductas, actitudes y valores relacionados con el fair play en deportistas jóvenes. Investigaciones en ciencias del deporte, (9), 37-87.

6. Cruz, J., Boixadós, M., Capdevila, L., Mimbrero, J., Torregrosa, M., Y Valiente, L. (1999). Evaluación del fairplay en el deporte profesional y de iniciación. Participación deportiva: perspectiva ambiental y organizacional, (24), 7-52. Recuperado de https://revistasdigitales.csd.gob.es/ index.php/ICD/issue/view/42

7. Cecchini Estrada, J. A., González González-Mesa, C., y Montero Méndez, J. (2007). Participación en el deporte y fair play. Revista anual de psicología Psicothema versión electrónica, 19(1), 57-64. Recuperado de http://www.unioviedo.net/reunido/index.php/PST/article/view/8597

8. De Marziani, F. (2008). Fútbol infantil:; Trabajo o diversión?̨ Esfuerzo o placer?: Competencia o juego? Pena o gloria?. Educación Física y Ciencia, 10, 29-44.

9. Gil-Madrona, P. (2006). Educar en valores a través de la práctica de juegos y deportes: elegancia en el talante, valores y actitudes asociados al deporte. Idea La Mancha: Revista de Educación de Castilla-La Mancha, 3, 152-159.

10. Ginesta Portet, X., (2007). Los valores en el deporte: una experiencia educativa a través del Barça-Madrid. Revista Comunicar, 15(28). 148 156. Recuperado de www.revistacomunicar.com

11. Gómez Mármol, A., Sánchez Martínez-Alcaraz, B. J., García Montiel,
J. A., López Sánchez, G. F., López Sánchez, L., \& De la Cruz Sánchez, E. (2011). Nivel de deportividad de tenistas y futbolistas. E-COACH: Revista Digital de Técnicos de tenis, 12, 37-42.

12. Gutiérrez Sanmartín, M. (1998). Desarrollo de valores en la educación física y el deporte. Apunts: educación física y deportes, ler trimestre(51), 100-108. Extraído de http://dialnet.unirioja.es/servlet/ articulo?codigo=297307 el 9 de marzo de 2014 .

13. López Sánchez, G. F., Ahmed, D., \& Díaz Suárez, A. (2017). Level of habitual physical activity among 13-year-old adolescents from Spain and India. A cross-cultural study. SPORT TK: Revista EuroAmericana de Ciencias del Deporte, 6(1), 67-74.

14. López Sánchez, G. F., Díaz Suárez, A., \& Smith, L. (2018). Analysis of body image and obesity by Stunkard's silhouettes in 3- to 18-year-old Spanish children and adolescents. Anales de psicologia, 34(1), 167-172. doi: 10.6018/analesps.34.1.294781

15. Postow, B. C. (1980). Women and masculine sports. Journal of the Philosophy of Sport, VII, 51-58.

16. Sánchez-Oliva, D.; Leo, M., F.M.; Sánchez-Miguel, P.A.; Amado, A., D. y García-Calvo, T. (2012). Antecedentes motivacionales de los comportamientos prosociales y antisociales en el contexto deportivo. Revista Internacional de Medicina y Ciencias de la Actividad Física y el Deporte. 12 (46). 253-270. Recuperado de Http://cdeporte.rediris.es/revista/revista46/artantecedentes $235 . \mathrm{htm}$

17. Snyder, E. E., \& Spreitzer, E. (1983). Change and variation in the social acceptance of female participation in sports. Journal of Sport Behavior, 6(1), 3-8.

18. Tejero González, C.M., (2014). Apuntes de clase, Resultados de la investigación sobre actividad físico-deportiva: tratamiento y análisis (Curso 2013/2014). Máster en innovación, evaluación y calidad en la Educación Física. Universidad Autónoma de Madrid. Facultad de formación para el profesorado. Madrid.

19. Velázquez, B., R., y Hernández, A., J.L., (2002). Deporte y formación de actitudes y valores en los niños, niñas y adolescentes de la comunidad de Madrid. Revista de Educación. Madrid, (331), 369-398. Recuperado de http://europa.sim.ucm.es/compludoc/AA?articuloId=222865 